Draft version August 13, 2019

Preprint typeset using $\mathrm{LAT}_{\mathrm{E}} \mathrm{X}$ style AASTeX6 v. 1.0

\title{
CORRELATIONS BETWEEN THE VARIATION OF THE IONIZING CONTINUUM AND BROAD ABSORPTION LINES IN INDIVIDUAL QUASARS
}

\author{
WEI-JIAN LU (陆伟坚) ${ }^{1}$ AND Ying-RU LIN (林樱如) ${ }^{2}$ \\ School of Information Engineering, Baise University, Baise 533000, China
}

${ }^{1}$ E-mail: william_lo@qq.com (W-J L)

${ }^{2}$ E-mail: yingru_lin@qq.com (Y-R L)

\begin{abstract}
We discover the significant (significance level of $>99 \%$ ) correlations between the fractional variation of the ionizing continuum and that of the CIV and/or Si IV BALs in each of 21 BAL quasars that have at least five-epoch observations from the Sloan Digital Sky Survey-I/II/III. This result reveals that the fluctuation of the ionizing continuum is the driver of most of these BAL variations. Among them, 17 show negative correlations and the other 4 positive correlations, which agrees with the prediction of photoionization models that absorption line variability response to ionization changes is not monotonic. 8 quasars out of 21 examples have been observed at least 30 times on rest-frame timescales as short as a few days, which reveals that changes in the incident ionizing continuum can cause BAL variability even in such a short period of time. In addition, we find that most of the 21 quasars show larger variation amplitude in Si IV than C IV, which reveals the ubiquity of saturation in these BALs (at least for C IV BALs).
\end{abstract}

Keywords: galaxies: active - quasars: absorption lines

\section{INTRODUCTION}

It is well known that the intrinsic absorption lines, including broad absorption lines (BALs; with line widths $>2000 \mathrm{~km} \mathrm{~s}^{-1}$; Weymann et al. 1991), narrow absorption lines (NALs; with line widths of only a few hundred $\mathrm{kms}^{-1}$ ) and mini-BALs (with line widths between NALs and BALs; e.g., Hamann \& Sabra 2004), can show variation in strength and shape over restframe timescales from days to years (e.g., Chen et al. 2015; Grier et al. 2015; Wang et al. 2015; He et al. 2017; Chen et al. 2018a,b; Hemler et al. 2019, and references therein). The intrinsic absorption line variability may be caused by ionization state variability of the absorption gas (e.g., Crenshaw et al. 2003, and references therein) or by variability in the coverage fraction that caused by, for example, gas transverse motion (e.g., Hamann et al. 2008). Investigation of the relationship between the variability of absorption lines and that of the continuum can provide clues in understanding variation mechanism of the absorption lines.

In recent years, many efforts in systematic studies on the intrinsic absorption lines have been made to confirm the relationship between the variability of absorption lines and that of the continuum. In the field of BALs and/or mini-BALs, a few works have reported the lack of correlation between the BAL and the continuum variations (e.g., Gibson et al. 2008; Wildy et al.
2014; Vivek et al. 2014). Based on a sample of 452 quasars from the Sloan Digital Sky Survey Data Release 10 (SDSS DR10), Wang et al. (2015) qualitatively confirmed that the variations of $\mathrm{CIV}, \mathrm{NV}$, and Si IV BALs/mini-BALs show high synchronization with those of the ionizing continuum and emission lines, and concluded that the variability of the absorption lines is driven mainly by variations in the gas ionization as response to the continuum variations. Based on the statistical analysis of a sample of 2005 BAL quasars from the SDSS-I/II/III, He et al. (2017) further concluded that variability of BAL in more than $80 \%$ of quasars are caused by the variation of the ionizing continuum. More recently, correlation between the variation of the ionizing continuum and BALs has been confirmed by several papers (Lu et al. 2018; Lu \& Lin 2018; Vivek 2019). In the field of NALs, Lu et al. (2017) confirmed, for the first time, the significant correlation between the variability of NALs and the ionizing continuum, using a twoepoch spectral sample of 40 quasars containing 52 variable C IV $\lambda \lambda 1548$, 1551 absorption doublets constructed by Chen et al. (2015). Chen et al. (2018a,b) further confirmed this correlation based on a larger variable NAL sample with more epoch observations.

Although the correlation between the the variability of intrinsic absorption lines and that of the ionizing continuum has been confirmed based on statistical analy- 
Table 1: The properties of 21 quasars and correlation results.

\begin{tabular}{|c|c|c|c|c|c|c|c|c|}
\hline \multirow{2}{*}{$\begin{array}{c}\text { Quasar (SDSS J) } \\
(1)\end{array}$} & \multirow{2}{*}{$\begin{array}{l}z_{\mathrm{em}} \\
(2) \\
\end{array}$} & \multirow{2}{*}{$\begin{array}{c}N_{\text {epochs }} \\
\text { (3) }\end{array}$} & \multirow{2}{*}{$\begin{array}{c}N_{\text {pairs }} \\
(4) \\
\end{array}$} & \multicolumn{5}{|c|}{ 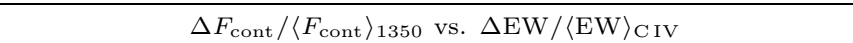 } \\
\hline & & & & $\begin{array}{c}r(p-\text { value })^{b} \\
(5)\end{array}$ & $\begin{array}{c}r_{\text {Bayes }}^{c} \\
(6)\end{array}$ & $\begin{array}{c}k_{\text {Bayes }}^{\mathrm{d}} \\
(7)\end{array}$ & $\begin{array}{c}b_{\text {Bayes }}^{\mathrm{e}} \\
(8)\end{array}$ & $\begin{array}{c}\sigma_{\text {int }}^{\mathrm{f}} \\
(9)\end{array}$ \\
\hline $022844.09+000217.0$ & 2.720 & 23 & 253 & $-0.37(0)$ & $-0.37 \pm 0.06$ & $-0.26 \pm 0.04$ & $0.07 \pm 0.01$ & 0.01 \\
\hline $023139.53+001758.3$ & 2.382 & 5 & 10 & $-0.78(0.0075)$ & $-0.85 \pm 0.18$ & $-2.22 \pm 0.74$ & $0.13 \pm 0.08$ & 0.03 \\
\hline $023252.80-001351.1$ & 2.030 & 20 & 190 & $-0.34(0)$ & $-0.32 \pm 0.07$ & $-0.40 \pm 0.09$ & $-0.02 \pm 0.02$ & 0.05 \\
\hline $024304.68+000005.4$ & 2.002 & 6 & 15 & $-0.59(0.021)$ & $-0.68 \pm 0.17$ & $-0.26 \pm 0.09$ & $-0.11 \pm 0.03$ & 0.01 \\
\hline $075007.63+275707.9$ & 2.365 & 5 & 10 & $-0.85(0.0016)$ & $-0.98 \pm 0.05$ & $-0.83 \pm 0.10$ & $0.03 \pm 0.03$ & 0.00 \\
\hline $140554.87+530323.7^{a}$ & 2.713 & 32 & 496 & $0.03(0.57)$ & $-0.02 \pm 0.05$ & $-0.02 \pm 0.05$ & $-0.01 \pm 0.01$ & 0.03 \\
\hline $141007.72+541203.6^{a}$ & 2.335 & 31 & 465 & $-0.56(0)$ & $-0.55 \pm 0.03$ & $-0.93 \pm 0.07$ & $-0.11 \pm 0.01$ & 0.02 \\
\hline $141421.54+522940.2^{a}$ & 2.050 & 32 & 496 & $-0.26(0)$ & $-0.38 \pm 0.04$ & $-0.13 \pm 0.01$ & $0.02 \pm 0.00$ & 0.00 \\
\hline $141955.28+522741.4^{a}$ & 2.145 & 32 & 496 & $-0.20(0)$ & $-0.29 \pm 0.06$ & $-0.92 \pm 0.22$ & $-0.02 \pm 0.02$ & 0.05 \\
\hline $142225.03+535901.9^{a}$ & 2.691 & 32 & 496 & $0.22(0)$ & $0.35 \pm 0.04$ & $0.22 \pm 0.03$ & $0.05 \pm 0.00$ & 0.01 \\
\hline $142404.66+532949.6^{a}$ & 2.768 & 31 & 465 & $0.39(0)$ & $0.47 \pm 0.04$ & $0.26 \pm 0.03$ & $0.00 \pm 0.00$ & 0.00 \\
\hline $142419.17+531750.8^{a}$ & 2.533 & 32 & 496 & $-0.36(0)$ & $-0.52 \pm 0.06$ & $-0.38 \pm 0.06$ & $-0.01 \pm 0.00$ & 0.00 \\
\hline $142422.50+525903.3^{a}$ & 2.142 & 30 & 435 & $0.01(0.89)$ & $0.08 \pm 0.07$ & $0.03 \pm 0.02$ & $0.02 \pm 0.00$ & 0.00 \\
\hline $164741.69+411545.3$ & 2.082 & 5 & 10 & $0.81(0.0049)$ & $0.56 \pm 0.27$ & $0.91 \pm 0.62$ & $0.26 \pm 0.09$ & 0.05 \\
\hline 004323.43-001552.4 & 2.799 & 7 & 21 & $-0.65(0.0016)$ & $-0.71 \pm 0.13$ & $-3.04 \pm 0.75$ & $-0.16 \pm 0.19$ & 0.36 \\
\hline $015048.83+004126.2$ & 3.697 & 5 & 10 & $-0.93(0.00011)$ & $-0.88 \pm 0.13$ & $-1.18 \pm 0.29$ & $0.03 \pm 0.07$ & 0.01 \\
\hline 022701.97-002621.7 & 2.295 & 20 & 190 & $-0.60(0)$ & $-0.57 \pm 0.05$ & $-1.20 \pm 0.13$ & $0.00 \pm 0.04$ & 0.29 \\
\hline $023233.57+000827.0$ & 2.659 & 19 & 171 & $-0.11(0.17)$ & $-0.09 \pm 0.09$ & $-0.24 \pm 0.24$ & $0.13 \pm 0.04$ & 0.18 \\
\hline 024557.23-000823.4 & 2.192 & 5 & 10 & $0.89(0.00054)$ & $0.80 \pm 0.19$ & $0.77 \pm 0.27$ & $-0.05 \pm 0.09$ & 0.03 \\
\hline 024747.59-004810.0 & 1.913 & 5 & 10 & $-0.90(0.00034)$ & $-0.07 \pm 0.59$ & $-3.12 \pm *^{\mathrm{g}}$ & $-0.12 \pm *^{\mathrm{g}}$ & 0.19 \\
\hline $234315.88+004659.5$ & 2.775 & 5 & 10 & $-0.77(0.0092)$ & $-0.74 \pm 0.25$ & $-0.13 \pm 0.07$ & $0.00 \pm 0.02$ & 0.00 \\
\hline
\end{tabular}

\begin{tabular}{|c|c|c|c|c|c|c|c|c|c|c|}
\hline \multicolumn{5}{|c|}{$\Delta F_{\text {cont }} /\left\langle F_{\text {cont }}\right\rangle_{1350}$ vs. $\Delta \mathrm{EW} /\langle\mathrm{EW}\rangle_{\text {Si IV }}$} & \multicolumn{6}{|c|}{$\Delta \mathrm{EW} /\langle\mathrm{EW}\rangle_{\mathrm{CIV}}$ vs. $\Delta \mathrm{EW} /\langle\mathrm{EW}\rangle_{\mathrm{SiIV}}$} \\
\hline $\begin{array}{c}r(p-\text { value })^{b} \\
(10)\end{array}$ & $\begin{array}{c}r_{\text {Bayes }}^{c} \\
(11)\end{array}$ & $\begin{array}{c}k_{\text {Bayes }}^{\text {d }} \\
(12)\end{array}$ & $\begin{array}{c}b_{\text {Bayes }}^{\mathrm{e}} \\
(13)\end{array}$ & $\begin{array}{l}\sigma_{\text {int }}^{\mathrm{f}} \\
(14)\end{array}$ & (15) & $\begin{array}{c}r(p-\text { value })^{b} \\
(16)\end{array}$ & $\begin{array}{c}r_{\text {Bayes }}^{c} \\
(17)\end{array}$ & $\begin{array}{c}k_{\text {Bayes }}^{\text {d }} \\
(18)\end{array}$ & $\begin{array}{c}b_{\text {Bayes }}^{\mathrm{e}} \\
(19)\end{array}$ & $\begin{array}{l}\sigma_{\text {int }}^{\mathrm{f}} \\
(20)\end{array}$ \\
\hline$-0.42(0)$ & $-0.40 \pm 0.05$ & $-0.30 \pm 0.04$ & $0.08 \pm 0.01$ & 0.01 & & $0.83(0)$ & $0.84 \pm 0.02$ & $0.79 \pm 0.03$ & $0.00 \pm 0.01$ & 0.00 \\
\hline$-0.95(0)$ & $-0.95 \pm 0.11$ & $-9.90 \pm 2.45$ & $0.14 \pm 0.28$ & 0.20 & & $0.92(0.0002)$ & $0.97 \pm 0.07$ & $0.24 \pm 0.04$ & $0.10 \pm 0.04$ & 0.01 \\
\hline$-0.39(0)$ & $-0.36 \pm 0.07$ & $-1.26 \pm 0.25$ & $-0.13 \pm 0.06$ & 0.44 & & $0.92(0)$ & $0.93 \pm 0.01$ & $0.32 \pm 0.01$ & $0.03 \pm 0.01$ & 0.01 \\
\hline$-0.80(0.00034)$ & $-0.77 \pm 0.14$ & $-1.08 \pm 0.28$ & $-0.05 \pm 0.10$ & 0.10 & & $0.71(0.0028)$ & $0.81 \pm 0.12$ & $0.23 \pm 0.05$ & $-0.10 \pm 0.03$ & 0.01 \\
\hline$-0.87(0.0012)$ & $-0.92 \pm 0.10$ & $-2.01 \pm 0.39$ & $0.10 \pm 0.12$ & 0.03 & & $0.84(0)$ & $0.96 \pm 0.05$ & $0.39 \pm 0.05$ & $0.00 \pm 0.03$ & 0.00 \\
\hline$-0.19(0)$ & $-0.25 \pm 0.05$ & $-0.33 \pm 0.06$ & $0.07 \pm 0.01$ & 0.05 & & $0.72(0)$ & $0.71 \pm 0.03$ & $0.54 \pm 0.03$ & $-0.05 \pm 0.01$ & 0.01 \\
\hline$-0.43(0)$ & $-0.44 \pm 0.04$ & $-0.72 \pm 0.07$ & $-0.08 \pm 0.01$ & 0.02 & & $0.83(0)$ & $0.85 \pm 0.01$ & $0.89 \pm 0.03$ & $-0.04 \pm 0.00$ & 0.01 \\
\hline$-0.62(0)$ & $-0.68 \pm 0.03$ & $-0.29 \pm 0.02$ & $0.00 \pm 0.00$ & 0.00 & & $0.55(0)$ & $0.73 \pm 0.02$ & $0.57 \pm 0.03$ & $0.03 \pm 0.00$ & 0.00 \\
\hline$-0.40(0)$ & $-0.60 \pm 0.06$ & $-3.75 \pm 0.50$ & $0.05 \pm 0.03$ & 0.15 & & $0.87(0)$ & $0.89 \pm 0.01$ & $0.45 \pm 0.01$ & $0.00 \pm 0.01$ & 0.01 \\
\hline $0.06(0.18)$ & $0.30 \pm 0.04$ & $0.28 \pm 0.04$ & $0.07 \pm 0.01$ & 0.01 & & $0.56(0)$ & $0.88 \pm 0.01$ & $0.60 \pm 0.02$ & $0.01 \pm 0.00$ & 0.00 \\
\hline$-0.40(0)$ & $0.47 \pm 0.04$ & $0.45 \pm 0.05$ & $0.02 \pm 0.01$ & 0.01 & & $0.64(0)$ & $0.70 \pm 0.03$ & $0.40 \pm 0.02$ & $-0.01 \pm 0.00$ & 0.00 \\
\hline$-0.25(0)$ & $-0.47 \pm 0.07$ & $-2.28 \pm 0.39$ & $-0.13 \pm 0.03$ & 0.12 & & $0.67(0)$ & $0.74 \pm 0.03$ & $0.11 \pm 0.01$ & $0.00 \pm 0.00$ & 0.00 \\
\hline$-0.46(0)$ & $-0.59 \pm 0.05$ & $-1.06 \pm 0.13$ & $-0.07 \pm 0.02$ & 0.03 & & $0.33(0)$ & $0.36 \pm 0.04$ & $0.07 \pm 0.01$ & $0.03 \pm 0.00$ & 0.00 \\
\hline $0.37(0.29)$ & $0.38 \pm 0.31$ & $0.54 \pm 0.60$ & $0.26 \pm 0.08$ & 0.05 & & $0.82(0.0038)$ & $0.95 \pm 0.07$ & $1.19 \pm 0.17$ & $-0.04 \pm 0.05$ & 0.01 \\
\hline$-0.26(0.26)$ & $-0.20 \pm 0.22$ & $-0.83 \pm 0.99$ & $-0.11 \pm 0.25$ & 0.66 & & $0.85(0)$ & $0.78 \pm 0.10$ & $0.87 \pm 0.17$ & $-0.45 \pm 0.13$ & 0.27 \\
\hline$-0.94(0)$ & $-0.98 \pm 0.06$ & $-3.80 \pm 0.58$ & $0.20 \pm 0.15$ & 0.02 & & $0.93(0.00011)$ & $0.92 \pm 0.11$ & $0.33 \pm 0.07$ & $-0.03 \pm 0.05$ & 0.01 \\
\hline$-0.74(0)$ & $-0.73 \pm 0.04$ & $-1.52 \pm 0.12$ & $-0.06 \pm 0.04$ & 0.20 & & $0.76(0)$ & $0.78 \pm 0.03$ & $0.79 \pm 0.05$ & $0.05 \pm 0.03$ & 0.17 \\
\hline$-0.46(0)$ & $-0.57 \pm 0.07$ & $-2.18 \pm 0.31$ & $0.29 \pm 0.05$ & 0.26 & & $0.69(0)$ & $0.77 \pm 0.04$ & $0.53 \pm 0.04$ & $0.03 \pm 0.02$ & 0.08 \\
\hline$-0.25(0.49)$ & $-0.27 \pm 0.34$ & $-0.49 \pm 0.83$ & $0.08 \pm 0.28$ & 0.23 & & $-0.12(0.75)$ & $-0.28 \pm 0.44$ & $-0.28 \pm 8.24$ & $0.12 \pm 0.24$ & 0.07 \\
\hline$-0.61(0.06)$ & $0.04 \pm 0.53$ & $*^{g}$ & $*^{g}$ & 0.14 & & $0.65(0.043)$ & $0.49 \pm 0.28$ & $0.71 \pm 0.53$ & $0.11 \pm 0.22$ & 0.22 \\
\hline$-0.76(0.011)$ & $-0.84 \pm 0.20$ & $-0.86 \pm 0.34$ & $-0.11 \pm 0.10$ & 0.01 & & $0.52(0.13)$ & $0.44 \pm 0.31$ & $0.09 \pm 0.08$ & $0.02 \pm 0.02$ & 0.00 \\
\hline
\end{tabular}

${ }^{\mathrm{a}}$ Quasars of the SDSS-RM project.

${ }^{\mathrm{b}}$ The Spearman rank correlation coefficient. The values in brackets are $p$-values, whose zero values represent $p$-value $<0.0001$.

${ }^{\mathrm{c}}$ The correlation coefficient from Bayesian approach (Kelly 2007).

${ }^{\mathrm{d}}$ The slope of the linear fit.

e The constant in the regression.

${ }^{\mathrm{f}}$ The variance of the intrinsic scatter.

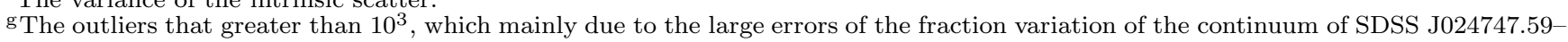
004810.0 . 
ses, the relationships between them in individual quasars are still unclear. As far as we know, only a few studies have reported correlation analysis between the variability of intrinsic absorption lines and that of the ionizing continuum in individual quasars with multi-epoch observations (Gabel et al. 2005; He et al. 2014; Huang et al. 2019). The large number of quasars with multi-epoch spectroscopic from the SDSS (York et al. 2000) provide a good opportunity for the research in this field. In this paper, we will investigate the relationships between the variability of absorption lines and that of the continuum in individual quasars with multi-epoch spectroscopic observations by SDSS. The sample selection and correlation analysis methods are given in Section 2. The discussions about our results are presented in Section 3. A brief conclusion is given in Section 4.

\section{SAMPLE SELECTION AND CORRELATION ANALYSIS}

Our initial sample consists of 2005 BAL quasars constructed by He et al. (2017) from the SDSS-I/II/III. Among them, there are 46 quasars have at least fiveepoch observations and a signal-to-noise ratio $(\mathrm{S} / \mathrm{N})$ level of $\mathrm{S} / \mathrm{N}>10$ in one-epoch observation at least. Because each quasar has $m(m \geq 5)$ spectra, there are a total of $C_{m}^{2}=m(m-1) / 2$ spectra pairs. We downloaded spectra of these 46 quasars from SDSS DR14 and fitted power-law continua for them using the procedure from Lu et al. (2018). We used the power-law continuum flux at $1350 \AA$ to represent the strength of the ionizing continuum for each spectrum. The equivalent width (EW) values of the BALs were derived from the catalog of $\mathrm{He}$ et al. (2017). Then we performed the correlation analyses between the fractional variation of the ionizing continuum and that of the CIV and/or Si IV BALs for each two observations of each the 46 quasars. Equations for calculating the fractional variation of the ionizing continuum and BALs is adopted from Lu et al. (2018) (equations (2) and (4) in therein). Finally, we got 21 out of 46 quasars showing significant correlations $(\mathrm{p}$-value $<0.01)$ between the fractional variation of the ionizing continuum and that of the CIV and/or Si IV BALs. Plots of the fractional variation of the ionizing continuum with that of the C IV and Si IV BALs, as well as the CIV versus Si IV BALs are shown in Figure 1. The results of the Spearman's rank correlation analysis are listed in Table 1.

\section{RESULTS AND DISCUSSIONS}

\subsection{The cause of the BAL variability}

The most important result in this paper is that we confirm the significant correlations between the fractional variation of the ionizing continuum and that of the C IV and/or Si IV BALs for each of 21 quasars. This is the first systematic study of the correlation analysis between the variability of intrinsic absorption lines and that of the ionizing continuum in individual quasars based on a large sample with multi-epoch observations. These statistical results serve as strong evidence for the idea that fluctuation of the ionizing continuum is the driver of most of the BAL variation of these quasars.

Among these sources, two have been investigated in details by previous papers. For one of them, SDSS J022844.09+000217.0 has been investigated by He et al. (2014). They found no significant correlation between the EW of CIV/Si IV BAL trough and the ionizing continuum in this source based on 18 epochs of spectroscopic observations, so they concluded that the BALtrough variability is not dominated by photoionization. However, we do find significant moderate anticorrelation between the fractional variation of the ionizing continuum and that of both CIV and Si IV BALs (see Table 1), when combining with five more epochs of spectroscopic observations (Table 1). Based on our results, we hold the view that the BAL-trough variation in SDSS J022844.09+000217.0 is dominated by photoionization, which is the response to the change of the ionizing continuum.

For the other one, SDSS J141007.74+541203.3 has been severally investigated by Grier et al. (2015) and Huang et al. (2019). Grier et al. (2015) found that (1) variations over the whole BAL trough rather than in some snippets, and (2) coordinate variability of the highvelocity CIV BAL (their Trough A) and the mini-BAL (their Trough B). Huang et al. (2019) reported the anticorrelation between the EW of BALs and the flux of the ionizing continuum of this quasar, based on just the spectra showing significant EW variations. Both Grier et al. (2015) and Huang et al. (2019) held the view that the most likely cause for the BAL variability in this source is a rapid response to the ionizing continuum changes. In this paper, we confirmed the significant anticorrelation between the fractional variation of the ionizing continuum and that of both CIV and Si IV BALs (see Table 1), which is consistent with the conclusion made by Grier et al. (2015) and Huang et al. (2019).

\subsection{Positive vs. negative correlations}

Another important result of our study is that we found both negative and positive correlation cases between the fractional variation of the ionizing continuum and that of the CIV and/or Si IV BALs. In fact, both the photoionization simulations (e.g., Wang et al. 2015; He et al. 2017) and systematic studies of the absorption lines (Wang et al. 2015; He et al. 2017; Lu et al. 2017; Chen et al. 2018b) have heralded that the ionic column density of a specific species could show response to a continuum variability positively or negatively. On the one 

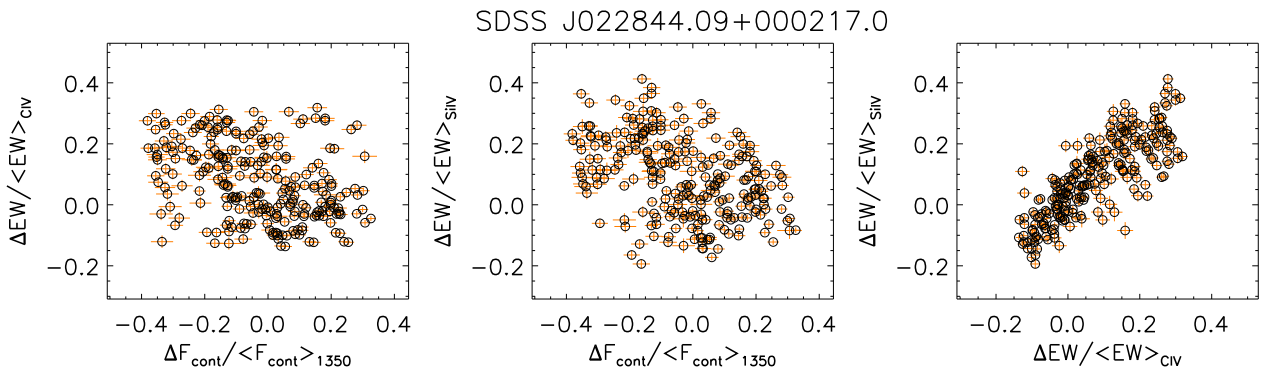

Figure 1: Plots showing the $\Delta \mathrm{EW} /\langle\mathrm{EW}\rangle_{\mathrm{CIV}}$ vs. $\Delta F_{\text {cont }} /\left\langle F_{\text {cont }}\right\rangle_{1350}$ (left panels), $\Delta \mathrm{EW} /\langle\mathrm{EW}\rangle_{\text {SiIV }}$ vs. $\Delta F_{\text {cont }} /\left\langle F_{\text {cont }}\right\rangle_{1350}$ (middle panels), and $\Delta \mathrm{EW} /\langle\mathrm{EW}\rangle_{\mathrm{Si} \mathrm{IV}}$ vs. $\Delta \mathrm{EW} /\langle\mathrm{EW}\rangle_{\mathrm{C} \text { IV }}$ (right panels) for each of 21 quasars.

hand, photoionization simulations show that absorption line response to ionization variability is not monotonic (see e.g., figure 3 in He et al. 2017). When the absorbing gas are at a relatively low ionization state, the EW of the $\mathrm{CIV} / \mathrm{Si}$ IV BAL show positive response to the continuum variation, otherwise, the negative response reveals the relatively high ionization state. On the other hand, based on large absorption line samples, statistical studies in both BALs (e.g., Wang et al. 2015; He et al. 2017) and NALs (Lu et al. 2017; Chen et al. 2018b) also showed that the variations of intrinsic absorption lines are positively or negatively responded to the alternations of quasar continua. These two kinds of respond suggest that the variable intrinsic absorption lines can be divided into at least two classes: one is highly ionized systems dominated by absorbing gas in high ionization and show negative response to changes of quasar emissions; and the other is lowly ionized systems dominated by absorbing gas in low ionization and show positive response to changes of quasar emissions.

However, rare convincing individual cases on the EW of $\mathrm{CIV} / \mathrm{SiIV} \mathrm{BAL}$ showing positive response to the continuum variation have been reported. Here, we find that 17 out of 21 examples show negative correlations, while 4 (SDSS J142225.03+535901.9, SDSS J142404.66+532949.6, SDSS J164741.69+411545.3, SDSS J024557.23-000823.4) out of 21 examples show positive correlations. Our results are consistent with the previous systematic studies and provide observational evidence for the photoionization model in explaining the variation of BALs.

\subsection{Rapid BAL variability}

8 out of our 21 examples were intensively observed in the year of 2014 in the Sloan Digital Sky Survey Reverberation Mapping Project (SDSS-RM; Shen et al. 2015), which obtained spectra of 32 epochs of 849 quasars on the SDSS 2.5m telescope (Gunn et al. 2006; Smee et al. 2013). In this project, the median spacing between observations is as short as about 4 days (Shen et al. 2015). Analyses on the time variability of BALs of the SDSSRM project were reported by Grier et al. (2015) and
Hemler et al. (2019). In this paper, we confirmed the significant correlation between the fractional variation of the ionizing continuum and that of CIV and/or Si IV BALs (see Table 1) in 8 quasars of the SDSS-RM project, which serve as strong evidence for ionization driven BAL variability as rapid response to changes in the incident ionizing continuum.

\subsection{Saturation effect}

Saturation in absorption lines can make significant influence on the correlation between the continuum and absorption line variations because a saturated absorber might respond to the continuum fluctuations softly or even no respond. Lu et al. (2018) pointed out that saturation in absorption lines could be one of the reasons for the substantial scatter of the plots for the fractional variation of the ionizing continuum versus that of both CIV and Si IV BALs. Lu et al. (2018) also found that the fractional variations of Si IV BALs seem greater than C IV BALs, which indicates that the C IV BALs suffering from more saturation than Si IV BALs. Lu \& Lin (2018) further confirmed the moderate anticorrelation between the Si IV BAL fractional variations and the ionizing continuum in 74 quasars that show obvious change in Si IV BAL but no or small change in C IV BAL (hereafter Phenomenon I), revealing the ubiquitous effect of the ionizing continuum variability on Phenomenon I. Vivek (2019) found that the BAL sample with shallow trough shows stronger correlation between the absorption line and the ionizing continuum variability.

Visually check Figure 1 we can find that most of the quasars show larger variation amplitude in SiIV than CIV, which is in agreement with previous studies. In particular, several quasars (e.g., SDSS J023139.53+001758.3, SDSS J142419.17+531750.8 and SDSS J142422.50+525903.3) show Phenomenon I. These results reveal the ubiquity of saturation in these BALs (at least for CIV BALs), however, the ionization variation in response to the ionizing continuum variations could still be the driver of their changes.

\subsection{Limitations}



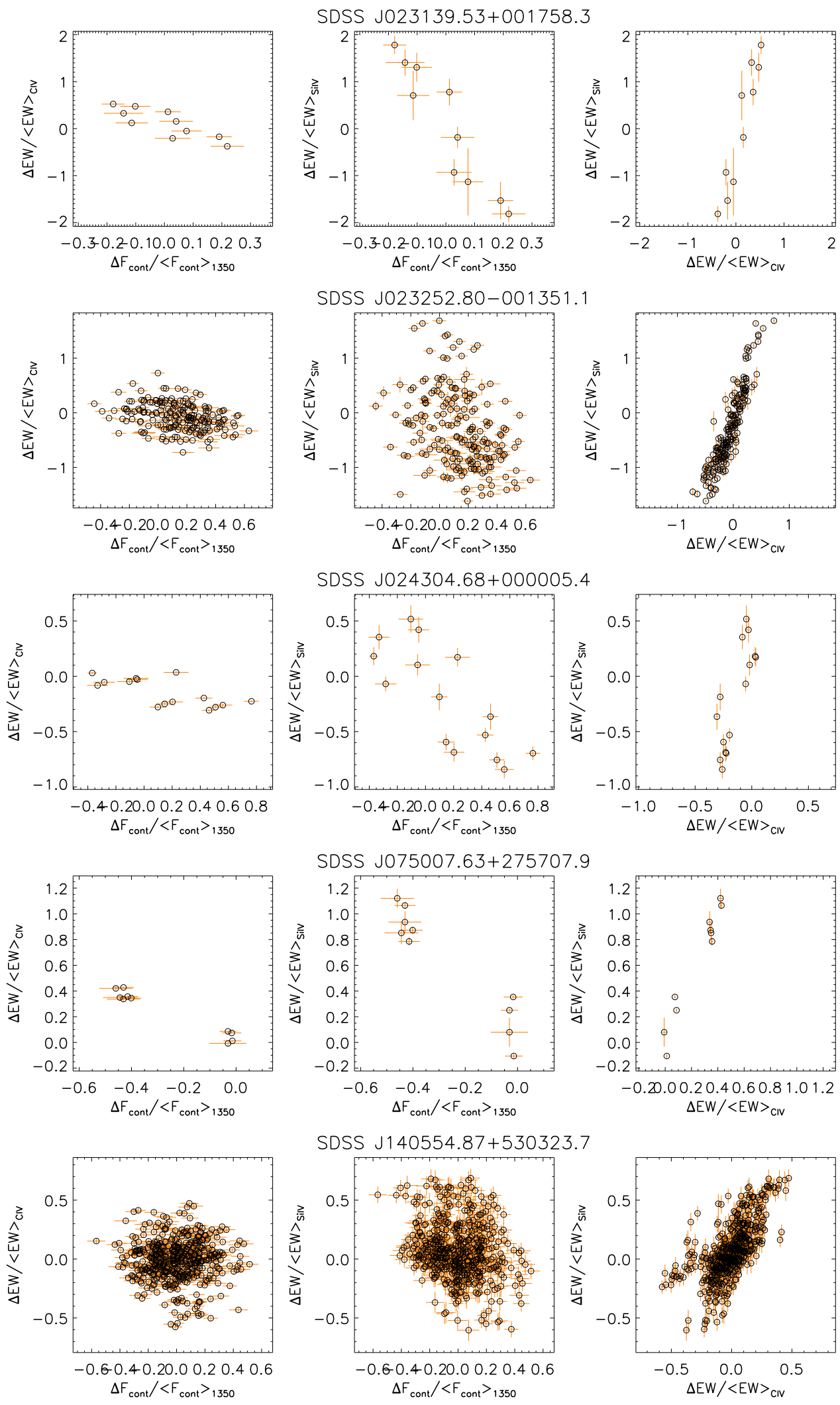

Figure 1 -continued 

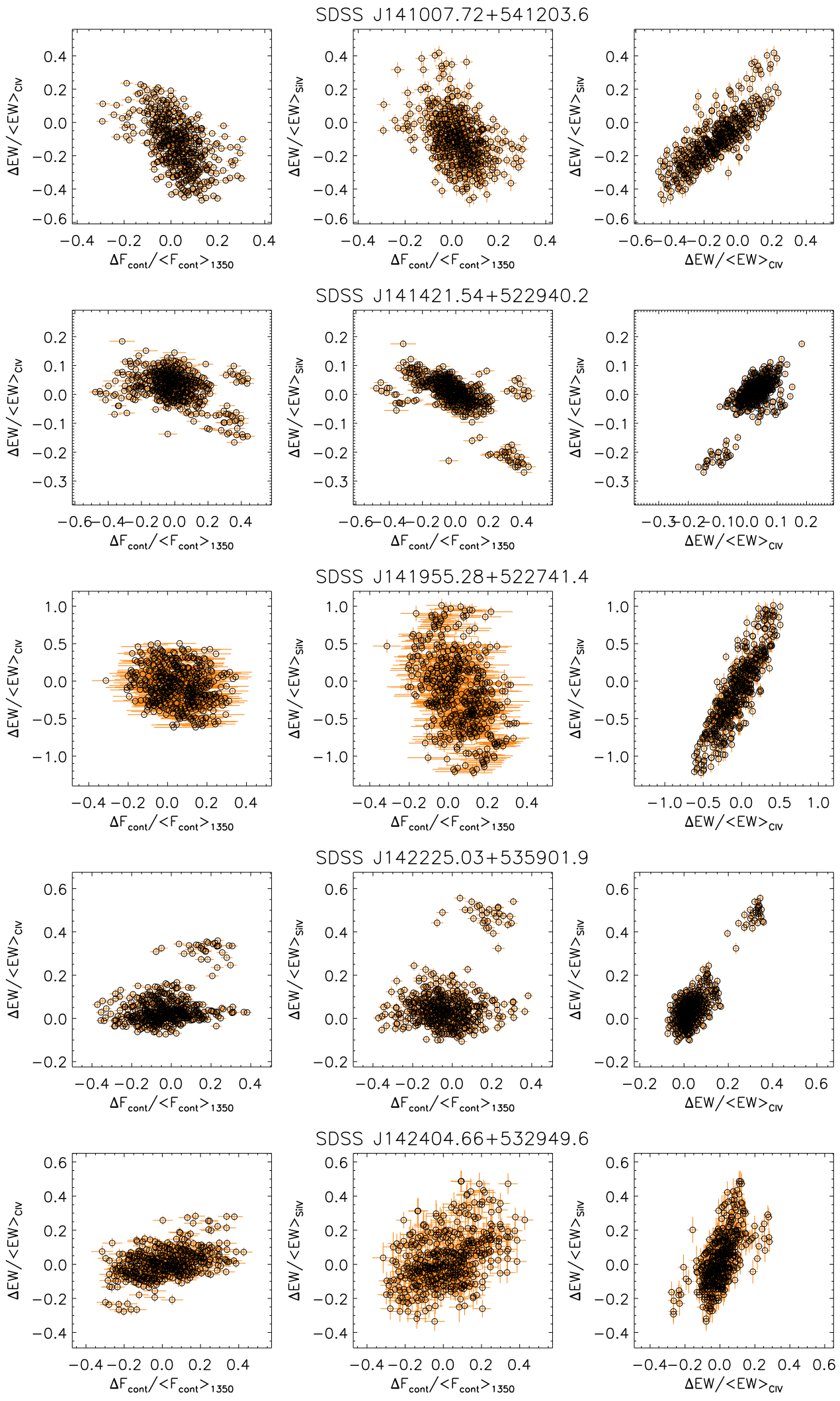

Figure 1 -continued 

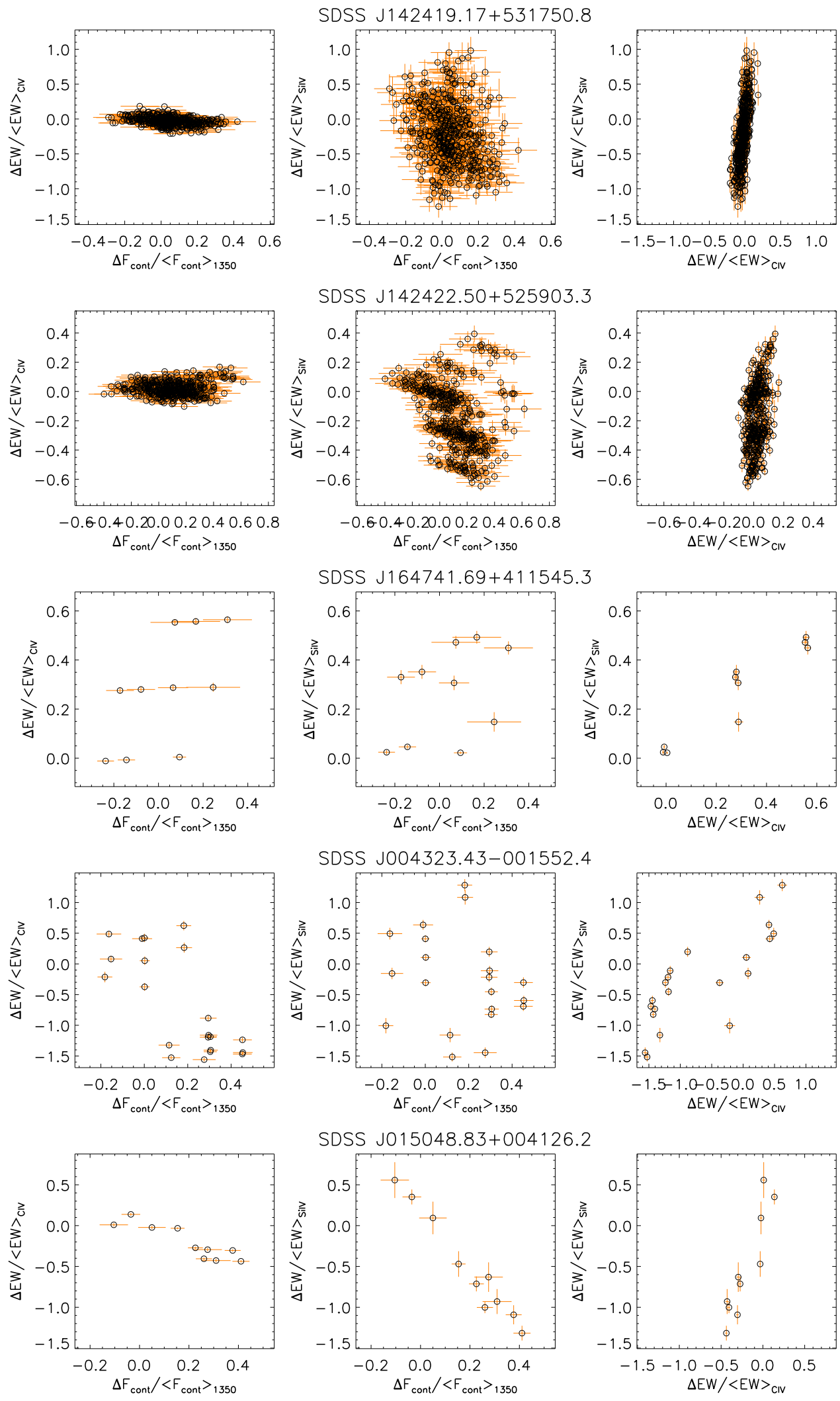

Figure 1 -continued 

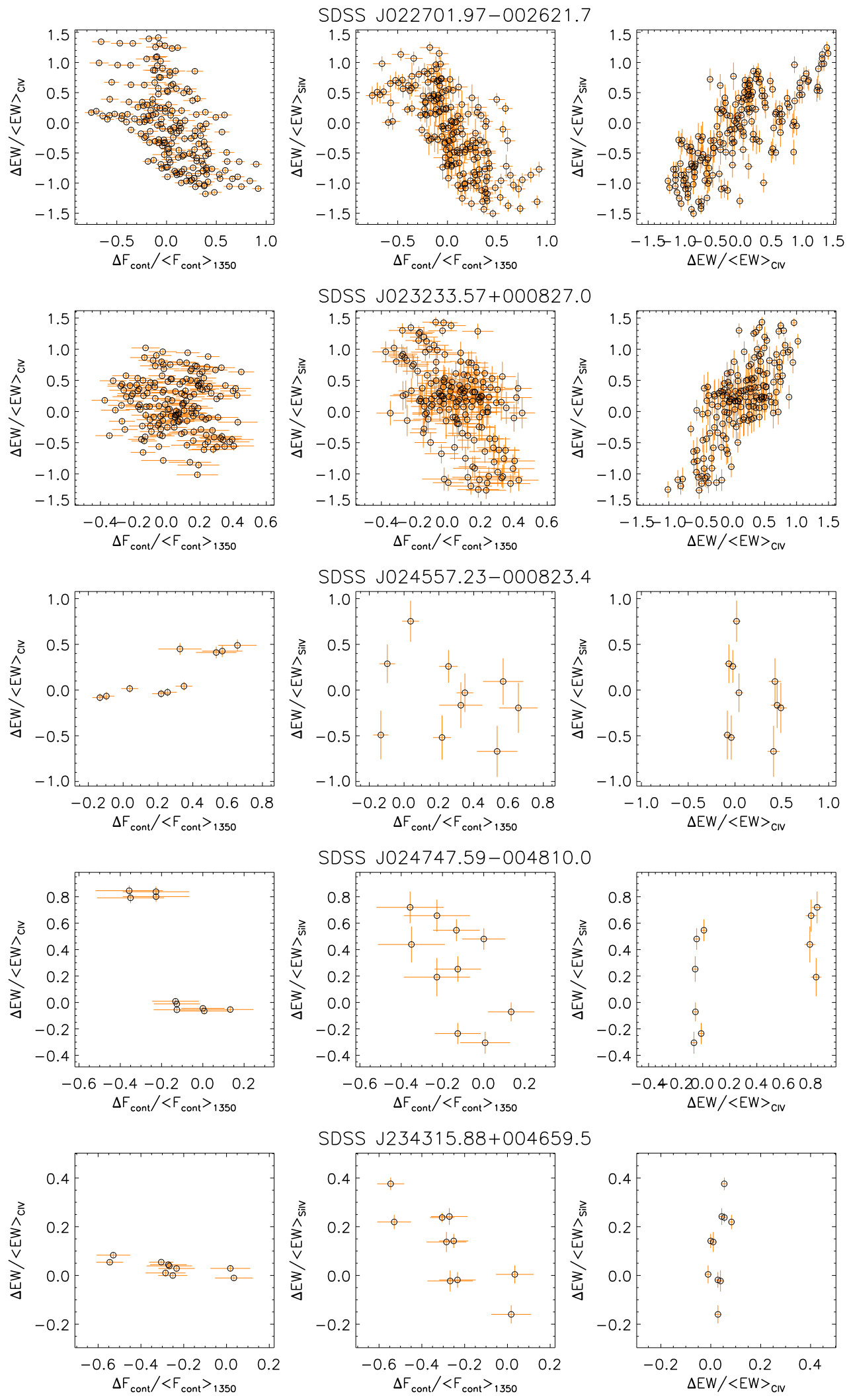

Figure 1 -continued 
Although the significant correlations between the fractional variation of the ionizing continuum and that of the $\mathrm{C}$ IV and/or Si IV BALs in each of 21 BAL quasars have been confirmed by the Spearman's rank correlation tests, most of the relations show substantial intrinsic scatter, which is corroborated by small correlation coefficients. Excepting the saturation effect from the BAL troughs (Section 3.4), the quality of the SDSS spectra (both resolution and $\mathrm{S} / \mathrm{N}$ ) may have a significant effect on this scatter. As a consistency check, we estimated the correlation coefficients and performed linear fittings using the Bayesian approach by Kelly (2007), which takes into account the measurement errors in the variables and intrinsic scatter. As shown in Table 1, most of the Bayesian results are consistent with the results of the Spearman's rank correlation analysis, which indicates that most of the reported correlations indeed hold. The only exception is the SDSS J024747.59-004810.0, which shows big deviation with large error. Future high quality monitoring will help us to confirm whether the correlation indeed hold or not in this quasar.

Another reason for the scatter could be that some quasars actually show no obvious EW variation in most of their observations. For example, the SDSS J142225.03+535901.9 and SDSS J141421.54+522940.2 both show obvious EW variation in only a few observations, which could be the reason of the gaps shown in the plots.

\section{CONCLUSION}

We have made the correlation analyses between the fractional variation of the ionizing continuum and that of the CIV and Si IV BALs for each of 46 BAL quasars that have been observed by SDSS at least 5 times, and have confirmed significant correlations in 21 of them. We have presented the following results and discussions on them.

(1) The significant correlations between the fractional variation of the ionizing continuum and that of the C IV and/or SiIV BALs for each of 21 quasars have been found. We think this result reveals the fluctuation of the ionizing continuum is the driver of most of these BAL variations.

(2) We find that 17 out of them show negative correlations, while the other 4 examples show positive correlations. These results support the previous systematic studies and the photoionization model, in which when the absorbing gas are at a relatively low ionization state, the EW of the C IV/Si IV BAL shows positive response to the continuum variation, otherwise, a negative response appears.

(3) 8 quasars out of our sample have been observed at least 30 times within a few days. The significant correlations between the fractional variation of the ionizing continuum and that of C IV and/or Si IV BALs (see Table 1) in these quasars serve as strong evidence for ionization driven BAL variability as rapid response to changes in the incident ionizing continuum.

(4) We find that most of the 21 quasars show larger variation amplitudes in Si IV than CIV, which reveals the ubiquity of saturation in these BALs (at least for C IV BALs). Saturation in BALs may lead to the substantial dispersion of the plots for the fractional variation of BALs. Even so, the correlations between the absorption line and the ionizing continuum variability still be apparently presented.

We are very grateful to the anonymous referee and the statistics editor for comments that improved the quality of this article.

Funding for the Sloan Digital Sky Survey IV was provided by the Alfred P. Sloan Foundation, the U.S. Department of Energy Office of Science, and the Participating Institutions. SDSS-IV acknowledges support and resources from the Center for High-Performance Computing at the University of Utah. The SDSS website is http://www.sdss.org/.

SDSS-IV is managed by the Astrophysical Research Consortium for the Participating Institutions of the SDSS Collaboration including the Brazilian Participation Group, the Carnegie Institution for Science, Carnegie Mellon University, the Chilean Participation Group, the French Participation Group, HarvardSmithsonian Center for Astrophysics, Instituto de Astrofísica de Canarias, The Johns Hopkins University, Kavli Institute for the Physics and Mathematics of the Universe (IPMU)/University of Tokyo, Lawrence Berkeley National Laboratory, Leibniz Institut für Astrophysik Potsdam (AIP), Max-Planck-Institut für Astronomie (MPIA Heidelberg), Max-Planck-Institut für Astrophysik (MPA Garching), MaxPlanck-Institut für Extraterrestrische Physik (MPE), National Astronomical Observatories of China, New Mexico State University, New York University, University of Notre Dame, Observatário Nacional/MCTI, The Ohio State University, Pennsylvania State University, Shanghai Astronomical Observatory, United Kingdom Participation Group, Universidad Nacional Autónoma de México, University of Arizona, University of Colorado Boulder, University of Oxford, University of Portsmouth, University of Utah, University of Virginia, University of Washington, University of Wisconsin, Vanderbilt University, and Yale University.

\section{REFERENCES}

Chen, Z.-F., Gu, Q.-S., Chen, Y.-M., \& Cao, Y. 2015, MNRAS, 450,3904 
Chen, Z.-F., Pang, T.-T., He, B., \& Huang, Y. 2018a, ApJS, 236, 39

Chen, Z.-F., Yao, M., Pang, T.-T., et al. 2018b, ApJS, 239, 23

Crenshaw, D. M., Kraemer, S. B., \& George, I. M. 2003, ARA\&A, 41, 117

Gabel, J. R., Kraemer, S. B., Crenshaw, D. M., et al. 2005, ApJ, 631, 741

Gibson, R. R., Brandt, W. N., Schneider, D. P., \& Gallagher, S. C. 2008, ApJ, 675, 985

Grier, C. J., Hall, P. B., Brandt, W. N., et al. 2015, ApJ, 806, 111

Gunn, J. E., Siegmund, W. A., Mannery, E. J., et al. 2006, AJ, 131,2332

Hamann, F., Kaplan, K. F., Rodríguez Hidalgo, P., Prochaska, J. X., \& Herbert-Fort, S. 2008, MNRAS, 391, L39

Hamann, F., \& Sabra, B. 2004, in Astronomical Society of the Pacific Conference Series, Vol. 311, AGN Physics with the Sloan Digital Sky Survey, ed. G. T. Richards \& P. B. Hall, 203

He, Z., Wang, T., Zhou, H., et al. 2017, ApJS, 229, 22

He, Z.-C., Bian, W.-H., Jiang, X.-L., \& Wang, Y.-F. 2014, MNRAS, 443, 2532
Hemler, Z. S., Grier, C. J., Brandt, W. N., et al. 2019, ApJ, 872, 21

Huang, H.-Y., Pan, C.-J., Lu, W.-J., et al. 2019, arXiv e-prints, arXiv: 1905.02407

Kelly, B. C. 2007, ApJ, 665, 1489

Lu, W.-J., \& Lin, Y.-R. 2018, ApJ, 862, 46

Lu, W.-J., Lin, Y.-R., \& Qin, Y.-P. 2018, MNRAS, 473, L106

Lu, W.-J., Lin, Y.-R., Qin, Y.-P., et al. 2017, MNRAS, 468, L6

Shen, Y., Brandt, W. N., Dawson, K. S., et al. 2015, ApJS, 216, 4

Smee, S. A., Gunn, J. E., Uomoto, A., et al. 2013, AJ, 146, 32

Vivek, M. 2019, MNRAS, 486, 2379

Vivek, M., Srianand, R., Petitjean, P., et al. 2014, MNRAS, 440, 799

Wang, T., Yang, C., Wang, H., \& Ferland, G. 2015, ApJ, 814, 150

Weymann, R. J., Morris, S. L., Foltz, C. B., \& Hewett, P. C. 1991, ApJ, 373, 23

Wildy, C., Goad, M. R., \& Allen, J. T. 2014, MNRAS, 437, 1976 York, D. G., Adelman, J., Anderson, Jr., J. E., et al. 2000, AJ, 120,1579 